\title{
Mind your language(s) \\ A discussion about languages and security
}

\author{
Éric Jaeger and Olivier Levillain \\ Agence Nationale de la Sécurité des Systèmes d'Information (ANSSI) \\ firstname.lastname@ssi.gouv.fr
}

\begin{abstract}
Following several studies conducted by the French Network and Information Security Agency (ANSSI), this paper discusses the question of the intrinsic security characteristics of programming languages. Through illustrations and discussions, it advocates for a different vision of well-known mechanisms and is intended to provide some food for thoughts regarding languages and development tools.
\end{abstract}

An unreliable programming language generating unreliable programs constitutes a far greater risk to our environment and to our society than unsafe cars, toxic pesticides, or accidents at nuclear power stations. Be vigilant to reduce that risk, not to increase it.

\section{C.A.R Hoare}

The French Network and Information Security Agency (ANSSI ${ }^{1}$ ) is an organism whose mission is to raise the security level of IT infrastructures to the benefit of governmental entities, companies as well as the general public. This includes identifying, developing and promoting methods or tools e.g. to improve correctness and robustness of software. In this area, the ANSSI has been conducting for a few years different studies on the adequacy of languages (including formal methods) for the development of secure or security applications. The objective of these studies was not to identify or specify the best possible language, but to understand the pros and cons of various paradigms, methods, constructions and mechanisms. Working with language specialists and industrial users, one of the first lessons learned was that there was no common understanding about this notion of intrinsic security of languages, and furthermore that some of the concerns of security experts were not understood at first by the other actors. This paper is intended to shed light on those concerns, as exhaustively as possible, through numerous illustrations. It attempts to summarise our journey among programming languages, identifying interesting features with potential impacts on the security of applications, ranging from language theory to syntactic sugar, low-level details at runtime, or development tools. Far from being conclusive, we expect no more than to promote, as far as security is a concern, alternative visions when dealing with programming languages; our professional experience is that this discussion is definitively useful today.

The subject of this article is therefore quite broad, and to some extent difficult to structure in an appropriate manner for the different communities. We have chosen to go from language theoretic aspects to low-level concrete details, then to discuss additional questions related e.g. to evaluation. After a short discussion about security, Sec. II considers abstract

\footnotetext{
${ }^{1}$ http://www.ssi.gouv.fr
}

features and paradigms of languages. We will then take a look under the hood, discussing syntax and more generally the concrete constructions provided by languages, that can have a noticeable impact on the quality of (or the confidence in) programs; this is the subject of Sec. III. Next, Sec. IV cast some light on those steps leading from source code to a running program that definitely deserve proper attention, such as the compilation phase and the execution environment. Once developed, a product has still to be tested and evaluated to derive an appropriate level of assurance, as it is discussed in Sec. V. Finally, Sec. VI ends this paper with a few lessons learned and perspectives.

\section{LANGUAGE SECURITY}

A secure software is expected to be able to cope with wilful aggressions by intelligent agents. In this sense, security approaches can significantly differ from functional ones (when the objective is e.g. to ensure that for standard inputs the software returns the expected results) or safety and dependability ones. In any case, bugs have to be tracked and eradicated, but securing a software may require additional care, trying to prevent unacceptable behaviors whatever the circumstances, regardless of their probability of occurrence. Consider for example a program for compression and decompression: the functional specification of Compress and Uncompress is that for any file $f$ we have Uncompress $(\operatorname{Compress}(f))=f$. However, it says nothing about the behavior of Uncompress when applied to arbitrary inputs, for example a maliciously crafted file, which is a common security concern (e.g. CVE-20100001 for gzip). We are also interested in security software, that is products offering security mechanisms. Such software has to be secure but has also to meet additional requirements to protect data or services. A good example discussed thereafter is a library providing cryptographic services, which is expected to protect the cryptographic keys it uses, preventing unauthorised modifications or information leaks ${ }^{2}$. Finally, we have to take care about the question of the evaluation. Indeed, a secure software may not be of any use in practice if there is no way to know that it is secure. It is therefore important to have processes to obtain appropriate assurance levels (see for example the Common Criteria standard [CC]).

There exist numerous works concerning language safety, and to some extent one may consider that languages such as ERLANG ${ }^{3}$ or $\mathrm{SCADE}^{4}$ have been designed specifically to cope

\footnotetext{
${ }^{2}$ Techniques used to protect cryptographic implementation against sidechannel attacks [CJRR99], [GP99] are out of the scope of this paper.

${ }^{3} \mathrm{http} / / /$ www.erlang.org

${ }^{4} \mathrm{http}: / /$ www.esterel-technologies.com/products/scade-suite
} 
with resilience, safety or dependability. Many general purpose formal methods can also be seen as tools to adequately address functional or safety needs. However, to our knowledge, the question of the security of languages is not so popular, and literature is still rather scarce. To be fair, there are books providing recommendations to develop secure applications in a given language, as well as proposals to natively include security features such as e.g. information flow control in languages [HA05]. But from our point of view, the security concerns are much broader than that, and should be addressed not only through coding recommendations or other software engineering methods, but also by due consideration during design phases of languages and associated tools. This has led ANSSI to conduct several works dealing with security and development, looking at several programming languages or development methods with a critical (and admittedly unfair) eye. This includes a first study to better understand the contributions of the JAVA language [ANS10], a second study on functional languages in general and OCAML in particular [ANS13], as well as a review of the benefits and limits of formal methods [JH09], [Jae10].

In the rest of this paper, we summarise many of our concerns through illustrations, to give some food for thoughts for the academic communities interested by languages. The sources of our concerns are numerous - and not always subtle: they range from syntax traps or false friends for inattentive developers to obfuscation mechanisms allowing a malicious developer for hiding a backdoor in a product while escaping detection by an evaluator, but also include theoretical properties not enforced in executable code or inappropriate specifications.

Code excerpts presented in the following sections use different programming languages. Our intent is not to criticise a specific language, but to illustrate concrete instances of our concerns (more often than not, the concepts are applicable to other languages). Furthermore, we may provide negative reviews for some mechanisms or concepts; the reader is expected to remember that we only deal in this paper with security concerns for critical developments, and that our messages should not be generalised too quickly to other domains.

Acknowledgement: We would like to mention that some of our illustrations have been derived from examples on different websites and blogs such as [Koi], [Atw], [FO] and (last but not least) [DW].

\section{ABSTRACT FEATURES AND PARADIGMS}

The tools we are trying to use and the language or notations we are using to express or record our thoughts, are the major factors determining what we can think or express at all!

\section{Edsger W. Dijkstra}

\section{A. Scoping and Encapsulation}

We start our discussion with a family of features existing in nearly any mainstream languages, namely the scoping of identifiers and the encapsulation. They are rather simple mechanisms, and such information is helpful e.g. for the compiler to define an appropriate mapping or to apply possible optimisations. Furthermore, they are attractive for the developer dealing with security objectives, e.g. to protect confidentiality or integrity of data. We believe that developers rely on many assumptions about frontiers between various parts of software - they expect proper separation between e.g. local variables of an application and a library it uses, and vice versa. Any mechanism blurring such frontiers is likely to translate into security concerns.

Variable scoping: In general, languages come with variables of different sorts, in terms of scope (local vs global) and life cycle (constant, variable or volatile). This is closely related to various theoretical concepts such as bound variables and $\alpha$-renaming, and is so common that it has become part of developers' intuition. But for a few languages, the design choices appear rather unexpected, especially when syntax does not provide any clue. Consider the following snippet in PHP:

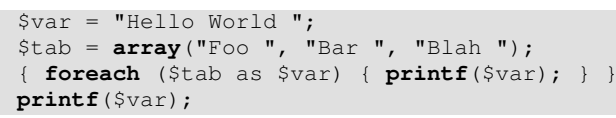

The foreach loop enumerates the values of the array \$tab and assigns them to the variable \$var. This code prints successively Foo, Bar, Blah... and Blah at its last line: the variable svar in the foreach loop is not locally bound and its previous value Hello World is overwritten. PYTHON has a similar behavior with the comprehension list construct, e.g. in $[s+1$ for $s$ in $[1,2,3]]$, that yields $[2,3,4]$, the variable s should be locally bound, but survives the definition. This is unexpected, and in fact inconsistent with other similar constructs in PYTHON such as the map construct ${ }^{5}$. This is, in our view, sufficiently unexpected and intriguing to be dangerous: developers rely on compositionality, and such poor managements of scopes mean that the semantics of closed - using only locally defined variables - pieces of code now depend upon their context.

Encapsulation: The encapsulation, e.g. for objectoriented languages, is a form of extension of scoping, but with a different flavor. In JAVA for example, one can mark a field as private to prevent direct access - except from other instances of the same class. Problems arise when a developer confuses this software engineering feature with a security mechanism. Consider this example in JAVA:

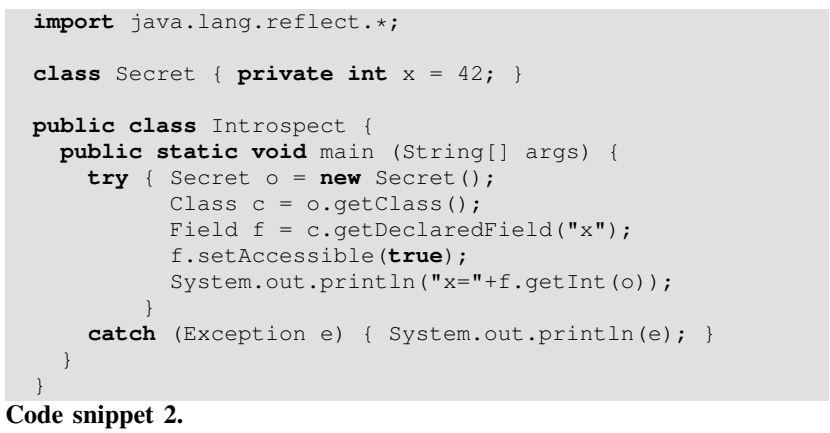

The printed result is $x=42$ : introspection is used to dynamically modify the class definition and remove the private mark. It is possible to forbid the use of introspection in JAVA with the so-called security monitor, yet this is a rather complex task - which is furthermore likely to have side effects on

\footnotetext{
${ }^{5}$ The behavior of comprehension lists has been fixed in PYTHON 3 but not in PYTHON 2, for the sake of backward (bugward?) compatibility.
} 
standard library services, as for example serialization in JAVA uses introspection.

OCAML provides encapsulation mechanisms through objects, but also a more robust version based on modules, whose public interface can be partial with respect to their actual implementation. For example, the following $\mathrm{c}$ module exports its id field but not key, as specified by its interface crypto:

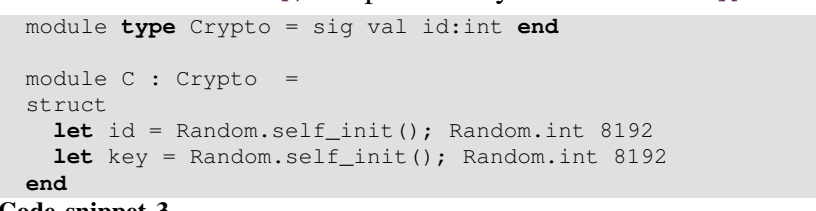

Code snippet 3.

That is, c.id is a valid expression whereas c.key is not, being rejected at compilation time. This is a pretty interesting feature to protect data, relying on well-studied type checking mechanisms: from the academic perspective, such a module is a closed box that cannot be open. Yet there are in OCAML literature descriptions that do not fit together. In [WL99], for example, a polymorphic function is described as a function that does not analyse the whole structure of its parameters, whereas the reference manual indicates that functions such as < are polymorphic and compare the structure of their parameters. Enters version 3.12 of OCAML, introducing first-class modules (i.e. modules are values that are comparable). This led us to write this little experiment:

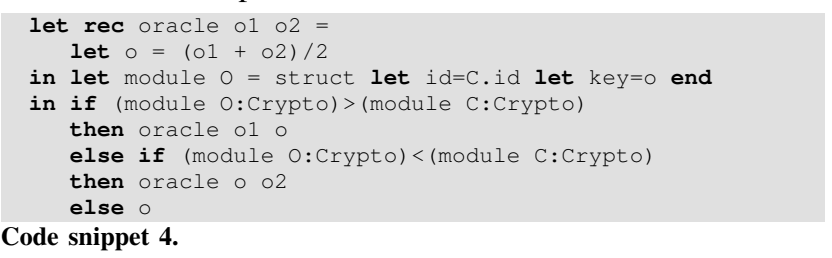

The function oracle is parameterised by $\circ 1$ and ${ }_{2}$, the lower and upper bounds. It creates a module $\circ$ with key set to $\circ$, the mean value of the bounds, and compare it with c. If o>c, the function is invoked again replacing the upper bound by $\circ$ (or the lower bound if $0<\mathrm{C}$ ). In practice, the oracle function finds the hidden value of c.key ${ }^{6}$. The attack is logarithmic in time but the main point is that we have been able to cross module frontier. Adopting again the academic perspective, the box has not been opened, but its contents is revealed by using a weighing scale. Some consider that this argue against polymorphic comparison operators in OCAML; for us it shows that trusted theoretical notions are not automatically and easily translated into robust security properties.

Our comments regarding encapsulation mechanisms are not intended to pinpoint errors in the languages: such mechanisms are convenient design tools of software engineering. On the other hand, it should be clear for developers that they are not, in general, robust security mechanisms.

\section{B. Side effects}

A fundamental result of typed $\lambda$-calculus (a pure functional language) states that evaluation strategy has no influence on the result of computations. Conversely, in presence of side

\footnotetext{
${ }^{6}$ The complete explanation of why it works also relies on the existence of a not-so-appropriate compiler optimisation.
}

effects, the order of computations may become observable. Provided this notion of evaluation strategy does not appear to be part of developer's common knowledge, we may expect some confusion when dealing with side effects. In [KR88] for example this is explicitly addressed by a clear and simple explanation of the difference of behavior between the \#define abs (X) $(X)>=0$ ? $(X):(-X)$ macro and the int abs (int $x)$ \{ return $x>=0$ ? $x:-x ;$ f function when one computes abs $(x++)$. Yet we would like to discuss much more intriguing situations:

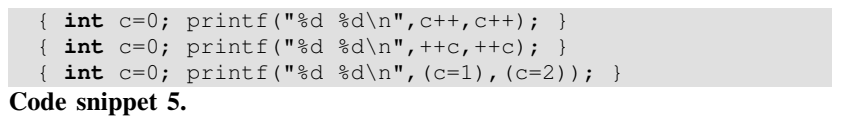

Well-informed $\mathrm{C}$ developers guess that the first line prints 10 as a consequence of right-to-left evaluation of parameters for the call-by-value strategy, but are generally surprised that the second line prints 2 2. At this stage, it would be cautious to admit that the pre- and post- increment operators can be too subtle to use. And, as in practice we will not miss them badly, why do they exist at all in the language? We are not over yet with side-effects, as affectations are by definition the primitive form of side-effect, and as a bonus are also a value in many languages such as $\mathrm{C}$ - a cause of troubles for generations of developers that have accidentally inserted an assignment instead of a boolean test in their if statements. The third line of the example therebefore prints 11 (this is also the final value of the variable $c$ ), something not so easily explained except by discoursing on calling conventions of $\mathrm{C}$. This type of code is confusing and explicitly discouraged by $\mathrm{C}$ standards: why then is it compiled without even a warning?

By extension, anything revealing the evaluation strategy can be considered as a side effect. Let us play again with macros and functions for simple tasks, what would be the difference between the macro \#define fst $(x, y) x$ and the function int fst (int $x$, int $y$ ) \{ return $x ;\}$ ? One can reveal which one is used with $f_{s t}(0,1 / 0)$, as the call-by-value strategy for functions will throw an exception. Pretty straightforward, so what do the following pieces of code?

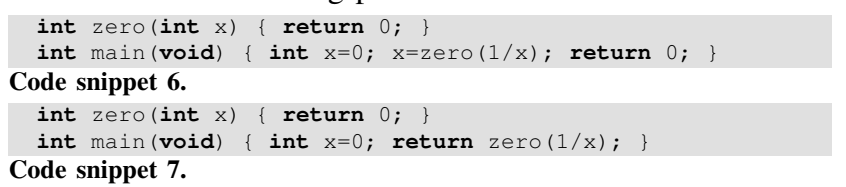

There is unfortunately no simple answer to this simple question: using the GCC compiler, there is an exception for both with option -00 , with option -01 the first code returns 0 and with option -02 the second also returns 0 . The fact that standard optimisations ${ }^{7}$ can modify the observable behavior of a program is worrying, and reveals a lack of clear and non ambiguous semantics for the $\mathrm{C}$ language.

As a last comment about side effects, let us go back to more basic observations but with unexpected applications to OCAML. In this language any standard variable is in fact a constant, in the sense that it can only be declared, allocated and initialised at once, and that it cannot be assigned later. Yet all allocations are managed by the garbage collector and variables live on the writable heap - this prevent the use of low-level security mechanisms such as storing constants in read-only

\footnotetext{
${ }^{7}$ Admittedly, the -03 flag is known to have curious effects and it's use is not recommended, but as far as we knew this was not the case with -02 .
} 
pages for example. OCAML also provides mutable strings (it is an impure functional language). Put together, this actually means that there is no way to have constant strings, which allows for dirty tricks such as this one:

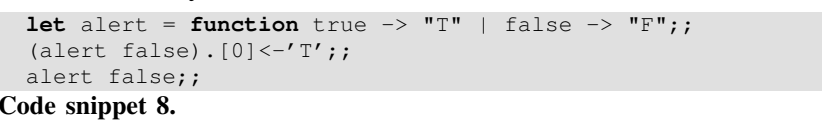

The first line of this code declares a function alert with a boolean parameter and returns a string, either " $\mathrm{T}$ " for true or "F" for false. The second line computes alert false to get the reference to the "F" string and overwrites its first char with ' $T$ '. As a consequence, the third line returns the string " $\mathrm{I}$ " instead of "F" - as any further invocation of alert false. Again, this is logical, yet surprising: as far as the developer is concerned, the source code of the function alert appears to have been modified by a side effect. This also applies to standard library functions of OCAML (at least in version 3.12 of the language ${ }^{8}$ ) and one can modify for example string_of_bool - by the way also impacting the behavior of Printf.printf. Similarly, it is a common practice in OCAML to parameter exceptions with strings, that can later be pattern-matched to take decisions; modifying such strings can then interfere with execution flow. As a final example, let us mention that the strings returned by char.escaped, which is a security mechanism, can also be meddled with.

\section{Types}

Don't you see that the whole aim of Newspeak is to narrow the range of thought? In the end we shall make thought-crime literally impossible, because there will be no words in which to express it. 1984, George Orwell

In Mathematics, type theory was a proposal of Bertrand Russel to amend Gottlob Frege's naive set theory in order to avoid Russel's paradox. In computer science, type checking is a well-discussed subject, and provides a good level of assurance with respect to the absence of whole categories of bugs - especially when the associated theory is proven. It can statically reject syntactically valid but meaningless expressions, but can also enforce encapsulation, manage genericity or polymorphism, and so on. In the family of ML languages [Mil84], [MT91], such as OCAML, it also leads to type inference and static verification of the completeness and relevance of pattern matching constructs, actually providing great assistance to developers. Type-checking is therefore relatively powerful, efficient and, last but not least, it does not contradict developers' intuition (yet advanced type systems may induce subtle questions, e.g. when dealing with subtypes and higherorder constructs [Pie02]). Thus we consider that type-checking is a must for secure developments, preferably static and strong to ensure early detection of ill-formed constructs.

Casts and overloading: It is often considered that strict application of type-checking concepts leads to cumbersome coding standards, not-so-friendly to developers. OCAML for example distinguishes between integer addition + and floatingpoint addition + . and does not automatically coerce values.

\footnotetext{
${ }^{8}$ Actions have been taken since then to avoid such manipulations e.g. by systematically returning copies of strings instead of the original ones.
}

The expression $1 .+2$ is therefore rejected, one correct version being 1.+. (float_of_int 2).

It is therefore standard for languages and compilers to ease developer's work by providing automated mechanisms allowing for overloading (using the same identifier for different operations) and automated casts or coercions. Yet we have various concerns about this approach. A first and trivial comment is that, whereas every student uses this type of trick, nearly none of them can explain what's going on. Casts and overloading are not a comfort anymore but a disguise.

All animals are equal, but some animals are more equal than others.

\section{Animal Farm, George Orwell}

But we are also worried by the consistency of design choices. Consider the example of ERLANG, in which expressions such as $1+1,1.0+1.0$ and $1+1.0$ are valid, implicitly inviting developers not to care about the difference between integers and floats. Let us now consider the typical example of the factorial function, presented in all beginner lessons:

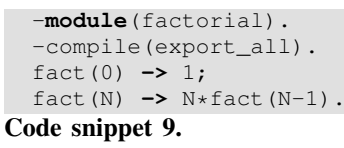

Without surprise, factorial:fact(4) returns 24, but on the other hand factorial:fact (4.0) causes a stack overflow. The same type of remarks apply to JAVASCRIPT, in which the condition $0==^{\prime} 0^{\prime}$ (comparing an integer with a string) is true, but a switch (0) does not match with case ' $0^{\prime}$.

Beyond such inconsistencies, one can also wonder whether casts and overloads are worth missing fundamental and intuitive properties. For example, equality is expected to be transitive, but this is not the case in JAVASCRIPT as ${ }^{\prime} 0^{\prime}==0$ and $0==^{\prime} 0.0^{\prime}$ are true but ${ }^{\prime} 0^{\prime}==^{\prime} 0.0^{\prime}$ is false. Similarly + can represents integer addition, floating point addition or string concatenation ; consider the following example:

$\mathrm{a}=1 ; \mathrm{b}=2 ; \mathrm{C}={ }^{\prime} \mathrm{FOO}^{\prime}$;

print $(a+b+c) ; \operatorname{print}(c+a+b) ; \operatorname{print}(c+(a+b))$;

Code snippet 10.

This code prints $3 \mathrm{~F}_{0} \mathrm{O}, \mathrm{F} 0012$ and Fo03. Whereas all the operations represented by + are associative, the property disappear for the composite (overloaded) operator.

Let us continue our discussion on casts and overloading with examples in the $\mathrm{C}$ language. Here again, this is notorious that unexperimented developers can be tricked, the canonical example being int $x=3$; int $y=4$; float $z=x / y$; which results in $z$ being assigned 0.0 . This one is maybe a little to easy to explain, so consider the following one:

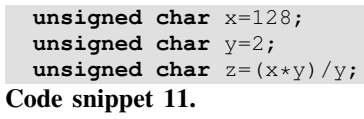

Some may be surprised to find out that $z$ is assigned 128 and furthermore disappointed that compiler's optimisation has nothing to do with this result: even in this code where all values have the same type, there are implicit casts. The cast mechanism in itself can be quite subtle too, as the following code prints $1>=-1$ and $1<-1$ :

unsigned char $\mathrm{a}=1$; signed char $\mathrm{b}=-1$;

if $(\mathrm{a}<\mathrm{b})$ printf $(" \circ \mathrm{d}<\% \mathrm{~d} \backslash \mathrm{n} ", \mathrm{a}, \mathrm{b})$; 


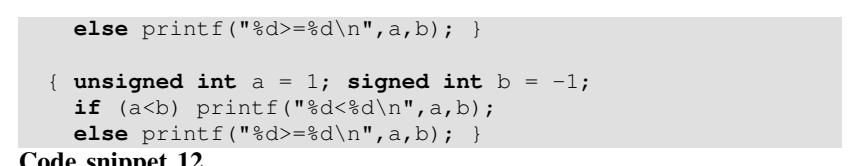

Code snippet 12.

At this stage, we have to consider coercions and overloading as false friends, on some occasions too devious to be managed properly. Are we too severe? Well, in practice, this leads to situations such as the following one:

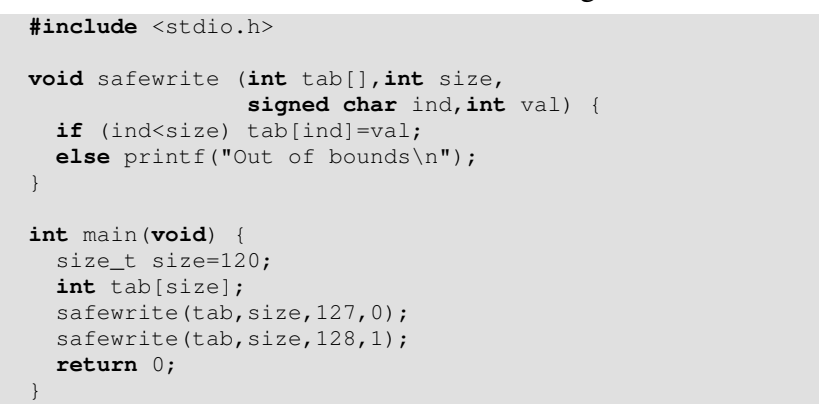

\section{Code snippet 13.}

safewrite $(t a b$, size, 127,0$)$ correctly produces the expected out of bounds message, but safewrite (tab, size, 128,0) succeeds - why and where the write occurs is left to reader's wisdom, as how would the program behave with size=150. Of course some compiler options (such as -wconversion for GCC) help to pinpoint such problems, but in practice there is a long history of bugs which are, in essence, similar to this one, leading to critical security vulnerabilities. For example, a really subtle bug in OpenSSL, CVE-2010-0740, was fixed by an even more subtle short patch:

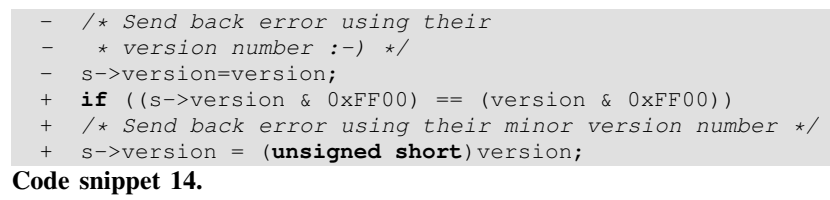

In the light of the previous examples, it might be hard to explain the exact consequences of adding an explicit integer cast, which can vary across architectures and compilers.

The JAVA language also allows developers for shooting themselves in the foot with overloadings as in this example, which prints $\mathrm{F} \circ \mathrm{O}, \mathrm{Bar}$ and $\mathrm{F} \circ \mathrm{\circ}$ :

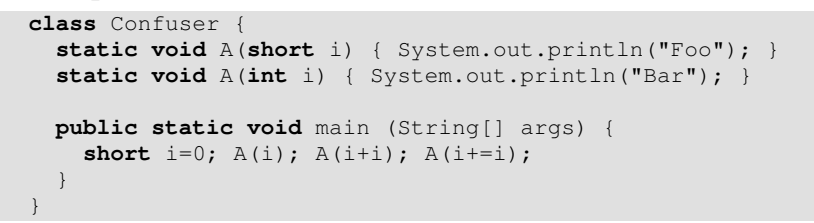

\section{Code snippet 15.}

PHP induces a whole new category of difficulties as far as types are concerned. One can play for example with string arithmetics, and increment with ++ a variable storing the string " $2 \mathrm{~d} 8 "$. The variable value is successively the " $2 \mathrm{~d} 9 "$ string, the "2e0" string and finally the 3 float, as "2e0" is interpreted as a scientific notation. The confusion between strings and numerical values is consistently reflected by the comparison operator $==$, which can induce casts even when comparing values of the same type. For example, the "0xf9"=="249e0" condition yields true as it does not compare the two strings but convert them respectively into int (249) and float (249). Let us illustrate the consequences of these mechanisms with the following piece of code:

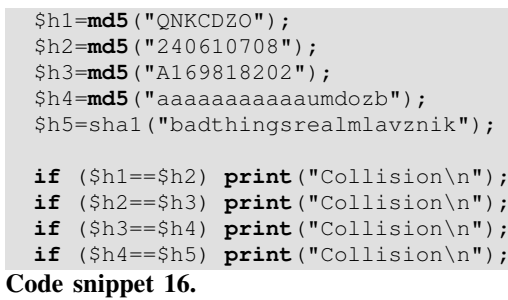

When executed, it prints collision four times. It is difficult to believe that we have indeed four distinct short strings with the same MD5 hash, and impossible to have a collision between an MD5 hash and a SHA1 hash. The trick is that each of the computed hash is itself a string matching the pattern $[0]^{+} e[0-9]^{+}$. When compared with $==$they are therefore all converted into the same value, that is float $(0)$.

To conclude this discussion, remember that we have presented type checking as a way to detect ill-formed expressions. To some extent, casts and overloading weaken this detection, the compiler being authorised to manipulate the code until it has a meaning. But one should avoid a situation in which any syntactically valid construct would become acceptable! JAVASCRIPT seems to be well advanced on this road, as all the lines in the following example are valid and have a distinct meaning, including the first and fourth one ${ }^{9}$ :

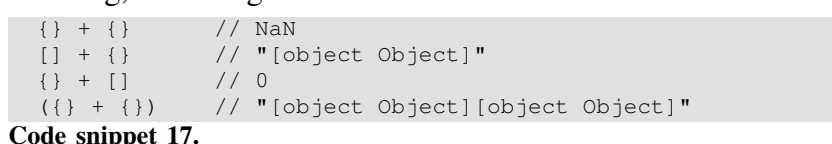

Type abstraction: Types provide a level of abstraction of programs convenient for the developers, but can lead to oversimplistic analyses in some cases. For example, one can consider that a well-typed boolean expression will return either true or false. We would argue however that other behaviors are possible and should be considered as well, for example looping computations or errors. Indeed, short-sighted view can easily become a cause of concerns, as illustrated here:

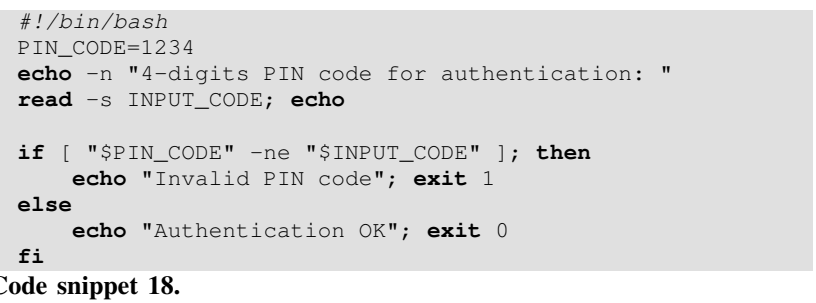

This script checks whether a PIN code is valid or not for authentication. One can conclude that if the provided code is 1234 , authentication will succeed, whereas any other value is rejected. However, in practice the test relies on a shell command which is expecting numerical values. Should a non numerical value such as blah be provided, this test would return an error code interpreted as being not true by the if statement, resulting in undue authentication.

In March 2014, an interesting vulnerability has been discovered in the GNUTLS library; let us just quote the analysis

\footnotetext{
${ }^{9}$ See http://jscert.org.
} 
provided on Linux Weekly News ${ }^{10}$ :

[This bug] has allowed crafted certificates to evade validation check for all versions of GNUTLS ever released since that project got started in late 2000.[...] The check_if_ca function is supposed to return true (any non-zero value in C) or false (zero) depending on whether the issuer of the certificate is a certificate authority (CA). A true return should mean that the certificate passed muster and can be used further, but the bug meant that error returns were misinterpreted as certificate validations.

Types at runtime: As a final remark about types, one should note that in static type-checking systems, types are pure logical information that have no concrete existence in implementations. This topic and its practical consequences are discussed in Sec. IV.

\section{Evaluators}

Some languages offer mechanisms to dynamically modify or create code, e.g. relying on evaluators. This is the case of the eval command in PHP, transforming strings into code which is executed. This allows for meta-programming and other dynamic features. As a related subject, we will discuss in Sec. IV the notion of attack by injection. For now, let us note that as far as security is concerned, the use of any form of evaluator makes a program impossible to analyse: in our view, language-embedded evaluators forbid security evaluation.

\section{SYNTAX AND SYNTACTIC SUGAR}

The elements presented in the previous sections deal with relatively high-level concepts. However, on occasions, the syntax of the language can be confusing or even misleading either for developers or evaluators. We provide a short review of concrete details that can become important.

\section{A. Consistency}

Come, let us go down and confuse their language so they will not understand each other.

Genesis 11:7

Identical keywords in different languages can have different semantics - something that we need to live with, but deserve adequate consideration e.g. for education of developers or evaluators. But it may be also that the same keyword or concept is used with several semantics in a language, depending upon the context. One can consider for example in JAVA the various possible meanings of the static tag, or the use of the interface concept as a flag to enable some mechanisms provided by the standard library (e.g. serializable). We find such design choices confusing and therefore potentially dangerous.

\section{B. No comments}

As noted in Sec. II, assignment $x=1$ is also an int value in $C$, whereas $x==1$ is a boolean condition (that is an int value). Confusing the two constructs is a common mistake, due to syntax similarity and the absence of warning from the compiler

\footnotetext{
${ }^{10} \mathrm{http}: / /$ lwn.net/Articles/589205/
}

using standard options. This can also be a trick used by a malicious developer, as in this now classical example ${ }^{11}$ of what appears to be an attempt to insert a Trojan horse in the LINUX kernel:

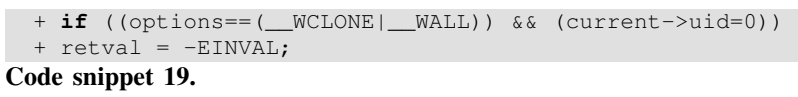

This small insert in the code of a system call mimics an error check. Yet in practice, should the two options _wCLONE and __WALL be set together (an irrelevant combination), and only in this case due to the lazy evaluation of $\& \&$, then the process user id would become 0 , that is root. This trap is discrete both with respect to syntax and behavior.

Don't get suckered in by comments, they can be terribly misleading.

\section{Dave Storer}

Some apparently irrelevant details of syntax may also be misleading to reviewers, e.g. the very basic concept of comments. For example in OCAML comments are surrounded by $(\star$ and $*)$, can be nested, but also have an intriguing feature. Consider the following piece of code:

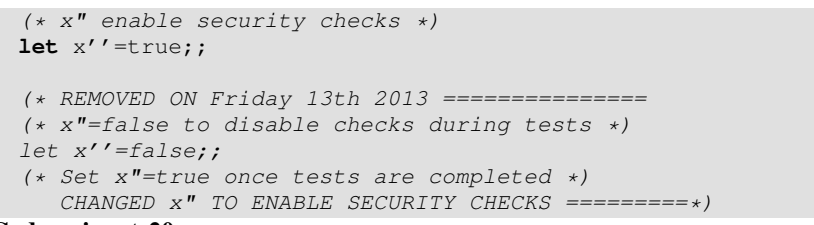

\section{Code snippet 20.}

At first, it seems that the line let $x^{\prime \prime}=$ true is executed, the rest of the code being commented out. But it is possible, in OCAML, to open a string in a comment - and this is exactly what we have done in our example. Therefore let $x^{\prime \prime}=$ true is in fact commented out, whereas let $x^{\prime \prime}=$ false is not. This is especially misleading when syntax coloring tools do not apply the appropriate rules (as this is indeed the case for some).

Similar tricks are possible in $\mathrm{C}$, such as in this example:

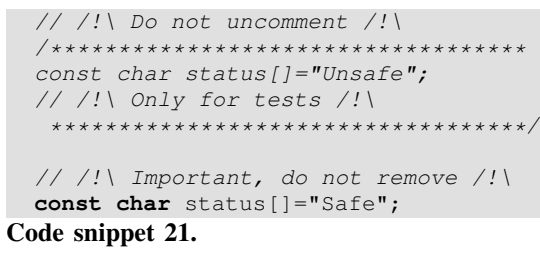

Of course, it assigns unsafe to the string status ${ }^{12}$. This can be used by a malicious developer to trick an evaluator, but it may also mean that, either because of a genuine ambiguity or because of misunderstandings, different tools can have different interpretations for the same source file, a perspective we are worried about.

\section{Encoding}

Let us conclude this section with a silly discussion about encoding. Some compilers allow for the use of UTF in source codes. We do not dispute the advantage of having many more symbols at hand, but we are definitely concerned by the

\footnotetext{
${ }^{11}$ lwn.net/Articles/57135.

${ }^{12}$ Provided there are no blank spaces after the trailing backslashes, which in itself is an interesting observation.
} 
numerous other possibilities offered by UTF-compliant tools. Consider this valid JAVA code:

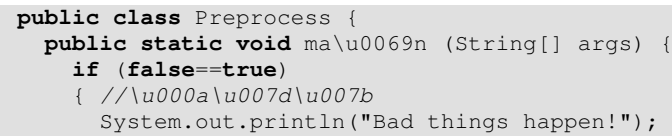

Code snippet 22

Despite appearances, from the JAVA compiler's perspective the method name is main, the comment mark is immediately followed by a line feed, a closing bracket and an opening one. The printin command, being out of the if block, is therefore executed. That is, using basic escape sequences, the structure of the code is modified. We have not yet investigated other interesting features such as right-to-left mark which allows for reverse printings (as well as overwrites), but we are definitively worried about them: there is a risk that different tools (such as the editor and the compiler in the previous example) provide different interpretations of the same source, leading to confusion or allowing for obfuscation.

\section{FROM SOURCE CODE TO EXECUTION}

Even when the programming language used to develop critical parts of a system offers all the desirable properties, what really matters from the evaluation perspective is the behavior of the program at runtime. Let us explore the long road from source code to execution, and some consequences for those desirable properties.

\section{A. Which program?}

Evaluators and interpreters: For critical systems, we have mentioned in Sec. II our concerns regarding the presence of evaluators in a language, as they make impossible analyses of programs. But we also need to mention that, by definition, such constructs allow for code injection. This applies to languages such as PHP, LISP that have built-in internal evaluators, but also to any other language which provides access to external evaluators.

Web applications are the canonical illustrations, transmitting strings interpreted as SQL queries by database engines. In PHP for example a common mistake is to build the query by concatenating constant strings with user-provided data, e.g. \$query="SELECT * FROM MyTable WHERE id $=$ '". \$login."' ", without realising what would happen should the variable \$login be assigned value "' OR $1=1$; DROP MYTable; -- ". But similar concerns exist e.g. when playing with exec-like features, using a shell as the interpreter, like system or popen in C.

There is no definitive conclusion about this type of vulnerabilities. Use of evaluators should be discouraged and submitted to thorough controls. It also justifies appropriate education for the developers, focusing not on recipes but on the principles, which are very generic: they apply not only to web applications in PHP but to any language with mechanisms blurring the frontier between data, metadata and code ${ }^{13}$.

\footnotetext{
${ }^{13}$ By the way, what would you expect as the result of the shell command $r m \star$ in a directory containing a file named $-\mathrm{f} r$ ?
}

Interpreted language: Various forms of code injections or unexpected executions are discussed in this paper. To palliate such vulnerabilities at system level, there are numerous known mechanisms and established practices. For example, one can control executable files, or rely on enforcing the $W \wedge X$ property to forbid a memory location to be both writable and executable. But what become such mechanisms with an interpreted language? As the code is only read and not executed (from the processor perspective), they offer no protection; the use of interpreted language therefore require an update of common security practices and thumb rules. Worse, with Just-In-Time (JIT) compilers, modern interpreted language need the memory to be writable and executable at runtime, preventing the use of $W \wedge X$ mechanisms, re-opening an avenue for attacks at the native level.

\section{B. Undefined behavior}

Discussing side effects, we have illustrated unspecified or inadvisable constructs that can be compiled and executed without warnings. There are other meaningless but supported constructs in $\mathrm{C}$, including very intriguing (and worrying) ones, such as for example pointer arithmetic applied to function pointers ${ }^{14}$. We have also indicated that in the absence of strong typing, some languages try and define the meaning of many possible expressions. A related problem in some languages is when the compiler leverages the presence of undefined behaviors to optimise the result.

For example, in $\mathrm{C}$, since dereferencing a null pointer makes no sense, the compiler can fairly assume that a dereferenced pointer is not null. Thus, in the following code, line 2 results in the assumption that tun is not null. The compiler can therefore remove the useless lines 3 and 4 for optimisation:

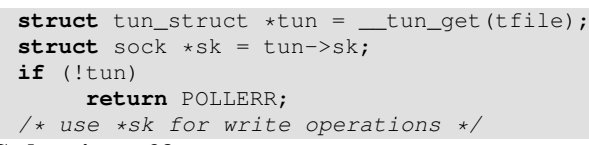

This particular code was part of the LINUX kernel in 2009 and led to a vulnerability (CVE-2009-1897). A recent study [WZKSL13] built on similar examples to analyse the interaction between undefined behaviors and compiler optimisations. However, some of these dangerous optimisations do not show in the source code, but are created as a result of previous intermediate steps, which makes it very hard to fix the general issue without drastically reducing the performance of the generated code.

\section{Encapsulation and compilation}

Many languages offer a form of encapsulation, but in general this abstract characteristic does not survive compilation, producing a single, monolithic memory mapping. This is understandable, as the disappearance of encapsulation has no observable effect on the execution of the program, whereas performance are as good as possible. Yet this also explain why in dysfunctional situations (such as error injection following a buffer overflow) there is usually no protection nor any detection mechanism.

\footnotetext{
${ }^{14}$ Yes, it works... Well, it compiles, executes, and does something
} 
JAVA, being executed as a bytecode on a JVM, emulate such protections - this is part of the job of the bytecode verifier. Of course, as mentioned in Sec. II, introspection can be a cause of concern, but let us put aside this question for a moment to consider a more intriguing question: JAVA allows for defining inner classes. Instances of the inner class have direct access to private fields of the containing class, and vice versa, as illustrated by this code:

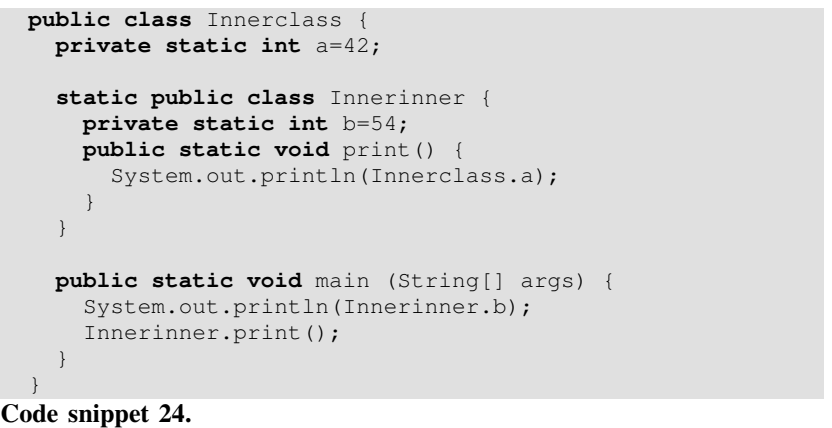

Interestingly, inner classes can be defined in JAVA but cannot be represented in bytecode - this is quite remarkable, as it means in theory that the JAVA language is more expressive than the JAVA bytecode and that the former cannot be compiled in the latter. Yet the previous example can be compiled, so how is it done? The inner class is in fact extracted and compiled independently. In order to maintain accessibility of both the outer and the inner classes to the a and $b$ fields, the private tags are removed. That is, those fields are now accessible from instances of other classes as well. In our view, such silent modifications of the code by the compiler should be banned.

\section{Memory protections in native binaries}

Usually, when the compilation phase produces a native executable, all the compilation units used are grouped in the same memory space, where only coarse grain protection can be enforced: read-only regions and non-executable stacks or data. As a matter of fact, the link between source code indications and binary memory mapping is sometimes not preserved, but can even be broken. Consider the following $\mathrm{C}$ code:

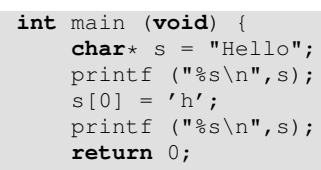

When compiling it on recent systems, the string literal " Hello" is put in the read-only data section at compile-time, leading to a segmentation fault when executing line 4 . The problem comes from the compiler that allows for a read-only string to be put into a read-write variable. This inconsistency vanishes when using $\mathrm{G}++$ instead of $\mathrm{GCC}$, or by adding in the options the -Wwrite-strings flag, which is off by default due to compatibility issues.

We believe that in some cases, it would be interesting to go further than the current implementations of compilers. Critical parts of a system could benefit from finer-grain memory protections. For example, in object-oriented languages, different classes (or even different instances of the same class) could be compiled as different processes or at least as different threads, relying on operating system mechanisms to provide stronger isolation. This would mean that the compilation steps also consider non-functional properties (like encapsulation) as invariants to be preserved along the way, whereas today they only care to maintain observable behaviors in standard conditions.

\section{E. About serialization}

In a static type-checking system, types are pure logical information which have no concrete existence in the actual implementation. It is a pity because very often types, being so intuitive, are implicitly used in developers' reasonings that is, some ill-typed situations may never be considered, whereas they can indeed occur. This is for example what make the wrap-and-decipher vulnerability of PKCS\#11 [Clu03] so elusive: the attack relies on exporting a cryptographic key which is later re-imported as a message. Keys and messages can indeed be confused in the physical world at execution time, whereas theoretical models can implicitly forbid such a scenario (see [JH09], [Jae10]). To some extent, this also explains why developers may omit to check the consistency of data in complex formats, e.g. trusting the provided uncompressed size field in the header of a compressed file, resulting in buffer overflow vulnerabilities when using $\mathrm{C}$-like languages.

Many modern languages provide an easy way for the developer to store and load binary objects as strings or files, the serialization. This mechanism allows for building complex structures (huge trees or hash tables) only once, saving them on disk and then relying on the deserialization to unfold the object without having to code a parser. In this context, our remarks about forgotten types apply. In practice, types are related to the interpretation method of such a string, and deserializing data is therefore often an act of faith, relying on the hope that the loaded string will be correctly interpreted as a value of the expected type. In general, modifying a serialized object on disk will lead to a memory error on loading, but the worst case scenario is to forge a serialized object directly pointing at memory cells it should not have access to. As a matter of fact, the LaFoSec study [ANS13] showed that the OCaml language was affected: during the deserialization, references between values are unfolded, yet no check insures the extracted references correctly point to deserialized values. It is therefore possible to forge a serialized blob containing pointers towards memory cells outside the blob - this is by the way the true meaning of the warning in the OCAML reference manual about Marshal.from_channel: Anything can happen at run-time if the object in the file does not belong to the given type. The same is true with the PYTHON pickle module, whose documentation explicitly recognises the presence of the vulnerability ${ }^{15}$.

On occasions, type information would be interesting to preserve at runtime as metadata - provided their integrity is ensured. But even when types are enforced at runtime, as it is the case in JAVA bytecode (without integrity protection, but this is not the subject of discussion here), serialization can still lead to serious security problems. Consider the following code:

\footnotetext{
${ }^{15} \mathrm{http}$ //docs.python.org/3/library/pickle.html states that [the] pickle module is not intended to be secure against erroneous or maliciously constructed data. Never unpickle data received from an untrusted or unauthenticated source.
} 


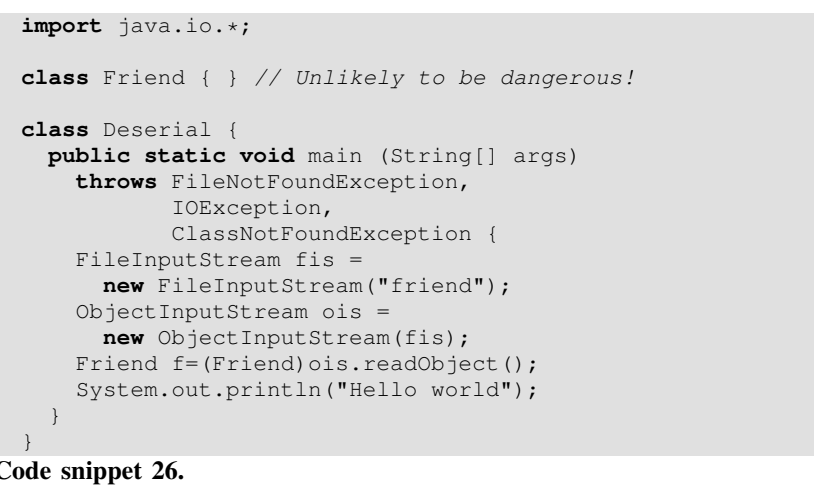

This code is intended to deserialize an instance of the class Friend, but this is not what happen in practice. Indeed, JAVA serialized files contains a reference to their class, and deserializing such a file automatically loads the corresponding class and executes its initialisation $\operatorname{code}^{16}$, before creating the instance in memory. It is later that this instance is casted possibly causing an exception, should types not be compatible. But this is far too late if the initialisation code is malicious! Thus JAVA serialized objects should not be seen as mere data. In 2008, a vulnerability was reported on the way the Calendar class deserialized foreign objects in a privileged context (CVE2008-5353). In this particular example it was shown that deserializing an object could lead to load a new class and to execute initialisation code with the privileges of the standard library classes.

\section{F. Low-level details matter}

To understand how some vulnerabilities operate in order to prevent or patch them, it is often necessary to understand how memory management works in a computer. Consider the following illustration of a format string attack:

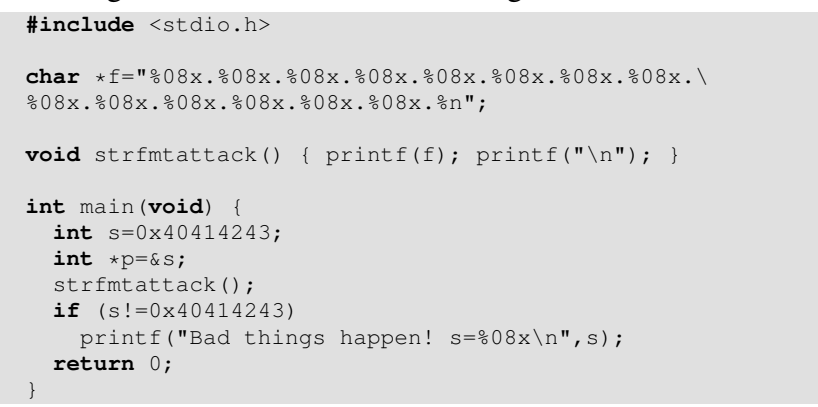

Code snippet 27.

strfmtattack uses printf to read and overwrite variable s which is outside its scope. But describing how stack buffer overflows or format string attacks work ([Ale96], [LC02]) requires knowledge on how variables, function arguments and return pointer are actually mapped in the memory. We certainly appreciate high-level programming languages and the features they provide for developers, such as automated memory management by a garbage collector preventing many critical bugs. On the other hand, we are not very comfortable with the related idea of teaching only high-level programming languages. Our fear is that if developers no longer need to call malloc and free because the language they use has a garbage

\footnotetext{
${ }^{16}$ That is code not included in a method and marked as static.
}

collector, there will be no point of teaching them what the stack and the heap are. This is plain wrong, as high-level languages in fine rely on native binaries and libraries. In particular, it ends up with this type of code ${ }^{17}$ :

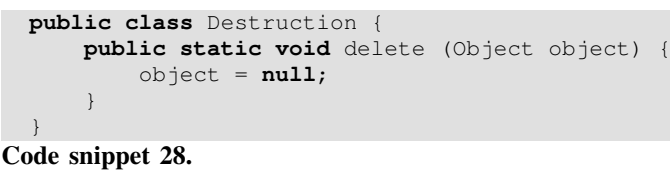

By the way, using a garbage collector to handle memory allocations instead of letting the programmer manage memory manually may have security drawbacks. Indeed, when a program manipulates secrets (passwords or cryptographic keys for instance), one has to minimise the duration of their presence in memory. He also has to ensure secure erasing by overwriting them with other values, so that they are no longer accessible via standard CPU instructions ${ }^{18}$. Those are common practice to tackle e.g. with swaps of memory pages, crashes or other situations in which the content of the memory is dumped and made accessible. Network devices exporting their secrets in a core dump after an easy-to-cause crash exist(ed).

Yet it is very hard (or even impossible) to control lifetime of a secret data in presence of a garbage collector. For the sake of performances, freed areas are generally not cleared by the garbage collector, for example. Some garbage collectors (e.g. the mark-and-copy flavor) might also spread the secrets several times across the memory.

Note that the same type of concerns may arise from other well-established mechanisms that have been developed to preserve observable behavior of programs but not other types of properties related to security. Erasure by overwritings, for example, can be removed by a compiler during optimisation (as there is no later readings), neutralised by cache mechanisms or flash memory controllers moving around physical writings to avoid fatigue of memory cells, and so on.

\section{ABOUT ASSURANCE}

Software and cathedrals are very much the same first we build them, then we pray

\section{Sam Redwine}

Developing correct and robust applications is difficult, and it has become even harder due to the increasing complexity of the manipulated concepts and systems. Furthermore, as mentioned in Sec. I, it is in general useless in industrial environments to have a secure software without knowing for sure that it is indeed secure. This also applies in other domains such as transportation systems, and leads to the notion of certification process, such as [IEC]. In IT security, the equivalent standards are the Common Criteria [CC], which define different evaluation assurance levels (EALs).

Security certification relies on analyses of the program and of its development process by independent evaluators, a process known as security evaluation. Discussing the intrinsic

\footnotetext{
${ }^{17}$ Source: http://thedailywtf.com/Articles/Java-Destruction.aspx; beyond the source code presented, many comments are worth reading to understand the level of culture regarding memory handling.

${ }^{18} \mathrm{We}$ are not talking here about physical attacks on memory chips or mass storage systems, so-called cold boot attacks, as described in $\left[\mathrm{HSH}^{+} 09\right]$.
} 
security characteristics of languages therefore requires appropriate consideration of the helps and limits that applies to the evaluation process. This includes for example an appreciation of the genuine understanding of both developers and evaluators of the features and mechanisms used. This is why we do not consider most advanced language constructs as fitted for critical developments, simpler approaches generally allowing for higher level of assurance.

There are two ways of constructing a software design. One way is to make it so simple that there are obviously no deficiencies. And the other way is to make it so complicated that there are no obvious deficiencies.

\section{C.A.R Hoare}

\section{A. About specifications}

In our view, developing an adequate level of mastery of the constructs of a language requires both practice and theory. The latter has not to rely on academic publications but can be addressed by proper specifications for the language, e.g. with well-defined semantics for its main constructs, including the standard library. But consider as a counter example this extract of the JAVA specification for the object class:

The general intent is that, for any object $\mathrm{x}$, the expression: $x . c l o n e() !=x$ will be true, and that the expression: $x \cdot c l o n e() \cdot \operatorname{getclass}()==x \cdot \operatorname{getclass}()$ will be true, but these are not absolute requirements. While it is typically the case that: $x$.clone().equals (x) will be true, this is not an absolute requirement.

In essence, it provides no requirements, and it would be unreasonable to expect anything about clone - and more so facing various implementations. This type of problem arises quite often, and various constructions in several languages appear to be only partially specified, voluntarily or not, explicitly or not. Let us consider for example two extracts of [KR88] for the $\mathrm{C}$ language:

The meaning of "adding 1 to a pointer" and by extension, all pointer arithmetic, is that pa+1 points to the next object, and pa+i points to the i-th object beyond pa.

The direction of truncation for / and the sign of the result for $\%$ are machine-dependent for negative operands, as is the action taken on overflow or underflow.

As already mentioned, the first extract appears relatively clear, even if it is informal; yet it says nothing about the expected behavior for function pointers. The second extract is a good example of a non deterministic specification: at least, the reader is explicitly informed that there is no guarantee about the result e.g. of $-1 / 2$.

It is worth mentioning at this stage that non-deterministic specifications can be tricky to handle. How would you test a compiler to check that the result of $-1 / 2$ complies with this specification? In general, the proposal is to check that it yields either 0 or -1 , but we would argue that this is not sufficient. Indeed, an implementation returning one or the other according to other parameters (e.g. time of the day or a secret value) would not be rejected. Yet you can be sure that having $-1 / 2$ not always equal to $-1 / 2$ (that is loosing reflexivity) would have consequences on the robustness of any software.

This is an instance of a problem known as the refinement paradox in formal methods such as B [Abr96]: given the nondeterministic specification $b \leftarrow$ getb $\triangleq b:=\top[] b:=\perp$ (that is getb is an operation returning a value $b$ which is either true or false), one often consider only the two constant solutions $b \leftarrow$ getb $\triangleq b:=\top$ and $b \leftarrow$ getb $\triangleq b:=\perp$. Yet there are other compliant implementations of getb in which the return value $b$ is still in $\{\perp, \top\}$ but depends upon dynamic values. This notion of refinement paradox ${ }^{19}$ applies to refinement-based methods such as B, but also to other formal methods such as COQ [Jae10].

As a summary, we prefer languages to be specified as completely, explicitly and formally as possible, avoiding non deterministic or partial properties. But we would also like to have tools such as compilers that provide errors and warnings when facing unspecified, discouraged or forbidden constructions.

\section{B. About code signature}

Code review by independent evaluators is a standard process in security evaluation. One has to question its relevance e.g. when dealing with object-oriented languages. In JAVA, accessing a variable can lead to dynamic loading and execution of code. Consider the following definition of a Mathf class:

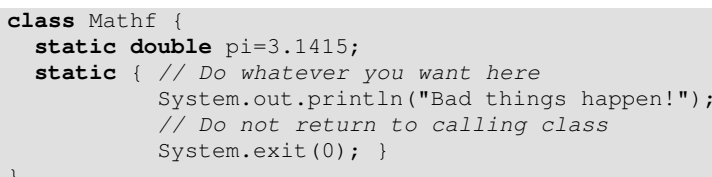

A JAVA program merely accessing Mathf.pi will execute the initialisation code of the Mathf class available on the system. The scope of the evaluation is therefore much broader, as nonexecutable files representing JAVA classes on the execution platform at runtime (rather than on the development platform at compile-time) have to be considered as well, as for the deserialization example of Sec. IV. This is one of the mechanisms supporting ad hoc polymorphisms in object-oriented languages; by comparison, the ADA genericity (with code specialisation at compilation) or the OCAML polymorphism (a single code for values of different types) are much more easy to manage.

In some cases, to compensate the lack of guarantees on the program which is actually executed, it is possible to rely on signature - provided signature checks cannot be avoided (and that public keys cannot be tampered with, that cryptographic protocols ensure required properties, etc.). It is usual for example in JAVACARD to verify the bytecode and sign the applet off-line, to avoid embedding a bytecode verifier in the card. However, defensive checks of source code properties (or proof-carrying codes) and code signatures have very different goals: the former aims at ensuring that a program behaves correctly whereas the latter only deals with its origin. In

\footnotetext{
${ }^{19}$ The term paradox is an overstatement, see e.g. [MM04].
} 
particular, code signing says nothing about the competence of the signer. All in all, code signing is useful for low-level libraries, when other defensive checks are not yet available. Here, the innocuousness of such code should be checked by source code audit and vulnerability analysis, signature only providing authentication on the execution platform (instead of any guarantee about the behavior of the code).

\section{The critical eye of the evaluator}

The different examples presented in the previous sections show that an evaluator should be aware of the numerous traps of programming languages.

When the evaluation process includes a source code audit, a good knowledge of the involved programming languages can help the evaluators, but we believe it is more important that they have a deep understanding of the features provided by the language and used by the developers. For example, objectoriented paradigm and serialization mechanisms are pervasive. The potential security issues are the same across programming languages implementing them. We have also discussed the very generic concept of code injection.

As we saw earlier, as the developer's intuition might be shattered by some constructions, it is also important to question the properties advertised by the language and their practical robustness: for example, checking that a private field is indeed not accessible may be rewarding, as in the JAVA inner class example discussed in Sec. II.

Finally, as illustrated in the Sec. IV, the evaluation of a product should depend on the intended final build process and the target runtime environment, since low-level details matter, and may vary from one platform to another. It is not rare to observe important differences between development and production builds, like the optimisation level; this may lead to different behaviors in practice, and even to real-world vulnerabilities as in the $\mathrm{C}$ undefined behavior case.

\section{About formal methods}

Beware of bugs in the above code; I have only proved it correct, not tried it.

Donald Knuth

At this stage, some of the readers may consider that the previous concerns raised would be solved by using deductive formal methods, allowing for proving program compliance with specifications, such as e.g. the B method [Abr96] or the CoQ proof assistant [Coq].

According to our analyses, this would be overoptimistic, as some of the problems pointed out still apply, and formal methods come with their own traps for inattentive developers or evaluators. This is discussed at length in [JH09], [Jae10], dealing with validity and completeness of the specification, the limits of expressivity of the formal languages, inconsistencies in the formal theory or bugs in the tools, trusted translations from formal to standard (executable or compilable) languages, and last but not least not satisfying explicit or implicit hypotheses when using proven software.

For example, in 2004 a variant of SSH was proven secure [Bel04], whereas in 2009 a plaintext-recovering attack was discovered [APW09]. In the former paper, Bellare et al. made an implicit assumption by working with binary streams already split into messages, whereas the attack presented indeed relied on this split operation.

\section{LESSONS LEARNED AND PROPOSALS}

We have considered numerous aspects of programming languages, trying to identify concerns when security is at stake. Rather than to attempt to provide definitive conclusions about which language should be used, we just provide a list with a few lessons that we learned during our journey, as well as some proposal for the way ahead.

\section{A. Languages and tools design}

First of all, as mentioned in the very beginning of this paper, we have been surprised to find that security of languages, when considered at all, was often so with a very narrow scope or wearing blinkers ${ }^{20}$. It is already common knowledge that designing languages is hard, in all likelihood an order of magnitude harder than learning and mastering a language. However, in the light of our work, some additional requirements should be taken into account.

If we certainly appreciate works related to more expressive type systems or the native integration of security mechanisms, we would like to avoid having feet of clay. This is why we advocate additional foundation works, defining which characteristics of languages are desirable to cope with security for critical developments. This requires considering common vulnerabilities or limits, as well as studies of how properties can be ensured or preserved from the theory to the real execution environment, and careful consideration of the robustness of the proposed mechanisms. We do not request new languages to be defined, but at least that evolutions of existing ones are discussed with some considerations about security in mind.

To add a few remarks about languages design when security is at stake:

- theoretical elegance must never go against developers' (or evaluators') intuition;

- eliminate non-determinism in the specification, and make explicit as far as possible undefined behaviors;

- always consider the advantages of new constructions but also the added complexity for developments or evaluations.

Beyond the language itself, attention should be payed to the associated tools, such as compilers, debuggers, or analyzers:

- avoid laxism, unspecified or undefined constructs should be tracked and signaled;

- ban silent manipulations;

\footnotetext{
${ }^{20}$ JAVA 8, published in March 2014, advertises on improved security, that is new cryptographic algorithms, better random generators, support for TLS, or PKCS $\sharp 11$ to cite a few. Yet we worry about the actual gains security-wise as these evolutions, in practice, may translate into additional vulnerabilities in an ever-more complex stack of codes and protocols build on sands.
} 
- consider new compilation invariants or instrumentation, e.g. to preserve type information, encapsulation, execution flow ${ }^{21}$;

- $\quad$ secure development tools should protect themselves against specifically crafted malicious inputs;

- $\quad$ ease inspection of actual program executed at runtime and traceability with source code;

- long-term goals should include the development of trusted (or certified) tools, such as the CompCert initiative [Ler11], [Dar09].

The robustness principle (also known as Postel's law) states that you should be conservative in what you do and liberal in what you accept. It aims at improving interoperability, but is not, in our view, appropriate when security is at stake.

\section{B. Training developers and evaluators}

Beyond messages addressed to language and tool specialists, we would also like to mention a few recommendations related to the education of developers - at least those dealing with critical systems and security concerns ${ }^{22}$ - which are of course also applicable to security evaluators.

First of all, developers should be ready, beyond functional approaches (checking that what should work works), to also adopt dual approaches (checking that what should not happen never happens). This includes for example worst-case reasonings related to unsatisfied pre-conditions, out-of-range values, ill-formed messages, etc. and can be supported by review of most common vulnerabilities and attacks.

Second, as attackers are always looking for the weakest link, developers should be able to have a broad vision encompassing most of the avenues of attacks. To this aim, we have the feeling that one has to learn the basics in several domains such as language semantics, compilation theory, operating system principles, computer architecture. These are, basically, the subjects that have been discussed in this paper when considering illustrations of our concerns.

\section{CONCLUSION}

In this paper, we presented some of the points we consider worth looking at while assessing the contribution of a language to the security of the programs written with this language, be they at the theoretical or the syntactic level, be they related to the compilation, the execution or the evaluation phase. This led us to propose some recommendations. We strongly believe that all the presented aspects should be taken into account when considering what languages bring to the security table.

\section{REFERENCES}

[Abr96] J.R. Abrial. The B-Book - Assigning Programs to Meanings. Cambridge University Press, August 1996.

\footnotetext{
${ }^{21}$ To cope with physical or logical fault injections.

${ }^{22}$ Quite often, IT security is expected to be managed only and independently by IT security experts through patches, intrusion detection systems, boundary protection devices, and so one. Yet we consider it is impossible to deal appropriately with security this way. Every developer should therefore be security-literate, in order not to introduce vulnerabilities to start with.
}

[Ale96] Aleph One. Smashing The Stack For Fun And Profit. Phrack, 49, 1996

[ANS10] ANSSI. Sécurité et langage Java. http://www.ssi gouv.fr/fr/anssi/publications/publications-scientifiques/ autres-publications/securite-et-langage-java.html, 2010.

[ANS13] ANSSI. LaFoSec : Sécurité et langages fonctionnels. http://www.ssi.gouv.fr/fr/anssi/ publications/publications-scientifiques/autres-publications/ lafosec-securite-et-langages-fonctionnels.html, 2013.

[APW09] M.R. Albrecht, K.G. Paterson, and G.J. Watson. Plaintext recovery attacks against SSH. In IEEE SSP, 2009.

[Atw] J. Atwood. Coding Horror. http://www.codinghorror.com/blog.

[Bel04] M. Bellare. Breaking and provably repairing the SSH authenticated encryption scheme: A case study of the encode-thenencrypt-and-mac paradigm. ACM TISS, 7:206-241, 2004.

[CC] ISO/IEC 15408: Common criteria for information technology security evaluation. http://www.commoncriteriaportal.org.

[CJRR99] S. Chari, C.S. Jutla, J.R. Rao, and P. Rohatgi. Towards sound approaches to counteract power-analysis attacks. In CRYPTO, pages 398-412, 1999 .

[Clu03] J. Clulow. On the security of PKCS\#11. In CHES, 2003.

[Coq] The Coq proof assistant. http://coq.inria.fr.

[Dar09] Z. Dargaye. Vérification formelle d'un compilateur pour langages fonctionnels. PhD thesis, Université Paris 7, 2009.

[DW] The daily WTF: Curious perversions in information technology. http://thedailywtf.com.

[FO] functional orbitz. http://functional-orbitz.blogspot.fr.

[GP99] L. Goubin and J. Patarin. DES and Differential Power Analysis The "Duplication" Method. In CHES, 1999.

[HA05] K. Hayati and M. Abadi. Language-based enforcement of privacy policies. In Privacy Enhancing Technologies, LNCS 3424. Springer, 2005.

$\left[\mathrm{HSH}^{+}{ }^{09}\right]$ J. Halderman, S. Schoen, N. Heninger, W. Clarkson, W. Paul, J. Calandrino, A. Feldman, J. Appelbaum, and E. Felten. Lest we remember: cold-boot attacks on encryption keys. Commun. ACM, 52(5):91-98, 2009.

[IEC] IEC 61508: Functional safety of electrical, electronic, programmable electronic safety-related systems. http://www.iec.ch/zone/fsafety/.

[Jae10] É. Jaeger. Study of the Benefits of Using Deductive Formal Methods for Secure Developments. PhD thesis, EDITE, 2010.

[JH09] É. Jaeger and T. Hardin. A few remarks about formal development of secure systems. CoRR, abs/0902.3861, 2009.

[Koi] S. Koivu. (Slightly) Random Broken Thoughts. http://slightlyrandombrokenthoughts.blogspot.fr.

[KR88] B.W. Kernighan and D.M. Ritchie. The C Programming Language. Prentice-Hall software series. Prentice Hall, 1988.

[LC02] K. Lhee and S.J. Chapin. Buffer overflow and format string overflow vulnerabilities. Software: Practice and Experience, 33:423-460, 2002.

[Ler11] X. Leroy. Verified squared: does critical software deserve verified tools? In 38th symposium Principles of Programming Languages. ACM Press, 2011. Abstract of invited lecture.

[Mil84] R. Milner. A proposal for standard ml. In LISP and Functional Programming, pages 184-197, 1984.

[MM04] A. McIver and C. Morgan. Abstraction, Refinement and Proof for Probabilistic Systems. Monographs in Computer Science. Springer Verlag, 2004.

[MT91] R. Milner and M. Tofte. Commentary on standard ML. MIT Press, 1991.

[Pie02] B.C. Pierce. Types and Programming Languages. MIT Press, 2002.

[WL99] P. Weis and X. Leroy. Le langage Caml. Dunod, 1999.

[WZKSL13] X. Wang, N. Zeldovich, M.F. Kaashoek, and A. Solar-Lezama. Towards optimization-safe systems: Analyzing the impact of undefined behavior. In Proceedings of the Twenty-Fourth ACM Symposium on Operating Systems Principles, SOSP '13, 2013. 